\title{
produits nouveaux
}

\section{Générateur d'aérosols calibrés "PLUS" (Pulvérisation Liquide UltraSonique)}

\author{
J. CHARUAU *
}

(Manuscrit reçu le 22 mars 1991)

\section{INTRODUCTION}

Le générateur "PLUS" crée des particules d'aérosols calibrés dans une large gamme granulométrique. Conçu pour une utilisation en laboratoire ou sur site, il ne requiert pas la compétence de spécialistes des aérosols. Cet outil nouveau permet de traiter expérimentalement de nombreux problèmes liés à l'aérocontamination.

Un aérosol est un nuage de particules liquides ou solides en suspension dans un gaz telles que brouillards, fumées..., d'origine naturelle ou industrielle. Bien que l'étendue granulométrique soit très vaste (de $10^{-3} \mu \mathrm{m}$ à $100 \mu \mathrm{m}$ ) l'essentiel du spectre se situe généralement entre 0,1 et $10 \mu \mathrm{m}$, cette dernière valeur étant considérée comme la limite des particules inhalables.

Dans le contexte de la radioprotection, la maîtrise de l'aérocontamination a nécessité le développement de techniques de simulation des particules contaminantes dans le domaine granulométrique observé lors d'incidents dans le cycle du combustible nucléaire. Ce besoin a conduit à l'élaboration du générateur d'aérosols calibrés "PLUS" dont la conception permet d'étudier in situ dans des laboratoires et usines :

- le transfert de la contamination dans les locaux,

- l'emplacement optimal des capteurs ou des points de prélèvement, - la rétention des particules dans les lignes de prélèvement, - l'efficacité de systèmes d'épuration ou de confinement dynamique, etc.

\footnotetext{
* Commissariat à l'énergie atomique, Institut de protection et de sûreté nucléaire, Département de protection de l'environnement et des installations, 91191 Gif-sur-Yvette Cedex.
} 


\section{PRINCIPE DE FONCTIONNEMENT}

La production d'un aérosol sec est obtenue à partir d'un traceur en solution ( $\mathrm{NaCl}$ par exemple). Cette solution est pulvérisée en brouillard qui, entraîné par un débit d'air sec, crée l'aérosol traceur voulu. La pulvérisation est réalisée à l'aide d'une technique ultrasonore dont le principe consiste à émettre des ondes de haute fréquence vers la surface de la solution. Ces ondes y provoquent une fontaine au sommet de laquelle se crée le brouillard. Cette technique présente, par rapport aux procédés habituels (pneumatique, par exemple), trois avantages importants:

- débit de génération élevé,

- variation aisée de ce débit,

- faible dispersion du spectre granulométrique.

Le diamètre des gouttelettes $d_{g}$ émises par la fontaine ultrasonore est constant car il dépend de la fréquence de résonance $(F)$ de la céramique piézoélectrique utilisée; il varie comme:

$$
\mathrm{d}_{\mathrm{g}}=\alpha \cdot \lambda \mathrm{c}
$$

où : $\alpha$ est une constante et,

ic la longueur d'onde capillaire est égale à :

$\lambda c=8 \pi\left[\frac{\sigma}{\rho}\right]^{1 / 3} \cdot F^{-2 / 3}$

Le diamètre des goutelettes ne varie qu'avec la fréquence $F$ pour une solution donnée de tension de surface $\sigma$ et de masse volumique $\rho$.

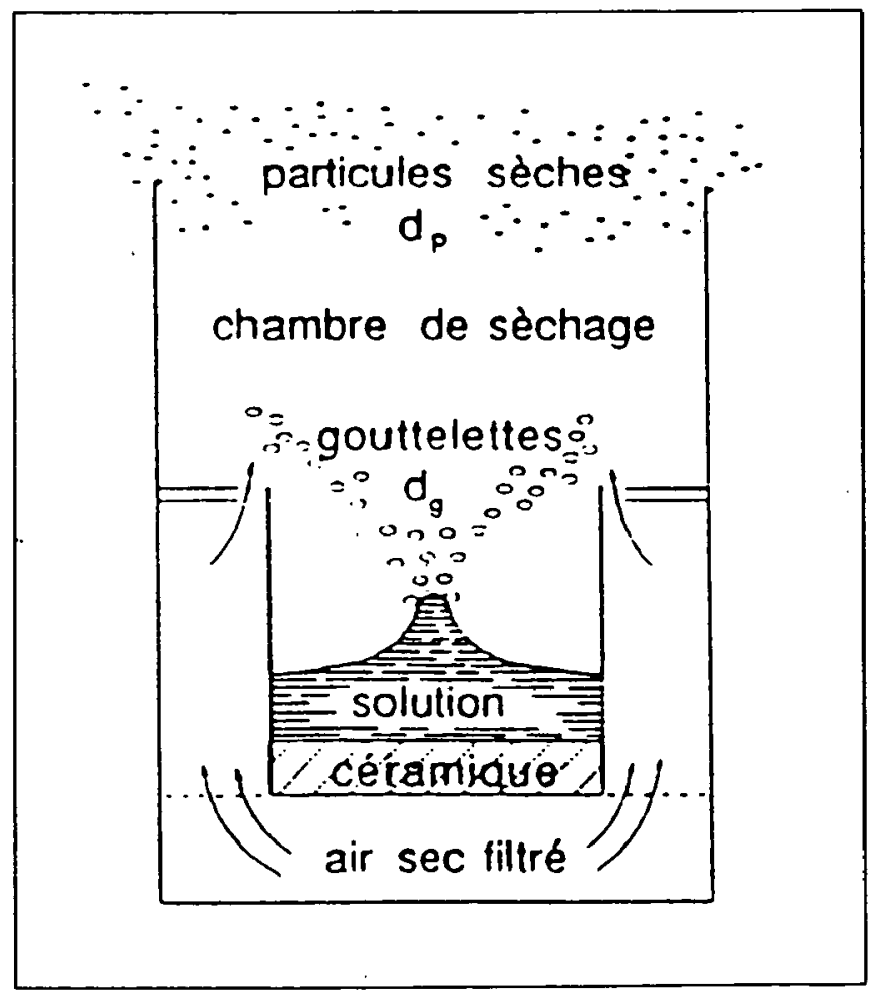


Après évaporation de l'eau des gouttelettes de diamètre $d_{g}$, on obtient des particules solides dont le diamètre $d_{p}$ dépend de la concentration volumique $\left(C_{v}\right)$ du traceur dissous, selon la relation:

$$
d_{p}=d_{g} \cdot\left(C_{v}\right)^{1 / 3}
$$

\section{PERFORMANCES DU GÉNÉRATEUR “PLUS”}

Pour couvrir l'ensemble des besoins déjà exposés, le générateur dispose de deux pots de pulvérisation fonctionnant l'un à $500 \mathrm{kHz}$ pour les particules les plus grosses, l'autre à $1800 \mathrm{kHz}$ pour les particules les plus fines. Un ensemble de capteurs de niveau et d'électrovannes permet le remplissage automatique de la solution à pulvériser qui est contenue

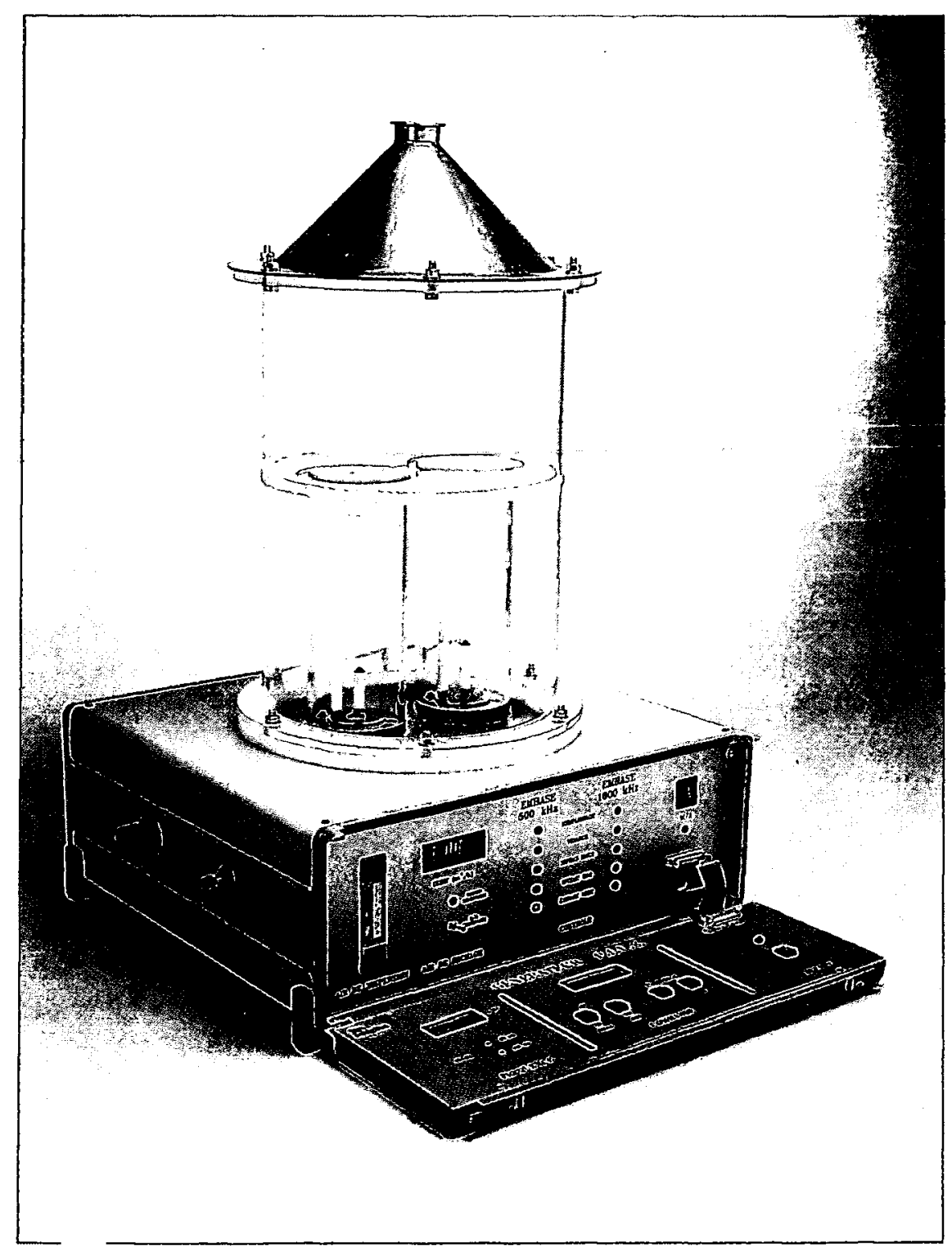


dans une réserve de volume adapté à la durée du fonctionnement désirée ; la vidange est également automatique. Le générateur, raccordé à une source d'air comprimé (réseau, bouteille ou compresseur) contient un circuit aéraulique complet qui permet l'alimentation et la mesure des débits d'air sec et propre. II contient également les circuits électroniques de conversion de fréquence, de commande de l'ensemble et de contrôle. Un microprocesseur gère les diverses fonctions, rend simple et conviviale l'utilisation de l'appareil.

II répond à des usages multiples parce qu'il offre la possibilité de choisir :

- la taille des particules d'aérosols entre 0,3 et $5 \mu \mathrm{m}$,

- le débit des aérosols secs entre $1 \mathrm{mg} / \mathrm{h}$ et $10 \mathrm{~g} / \mathrm{h}$,

- le débit d'air porteur entre 3 et $18 \mathrm{~m}^{3} / \mathrm{h}$.

Ces qualités en font le premier générateur d'aérosols calibrés disponible sur le marché dans cette gamme granulométrique et défini pour les besoins de la protection vis-à-vis de la contamination atmosphérique particulaire.

\section{EXEMPLE D'APPLICATION DE L'APPAREIL}

La mesure des coefficients de transfert d'un aérosol contaminant dans un local s'effectue à l'aide du générateur, qui simule le terme source, et de détecteurs spécifiques du traceur utilisé. Ces détecteurs réalisent la mesure de la concentration atmosphérique du contaminant (C en $\left.\mathrm{kg} \cdot \mathrm{m}^{-3}\right)$ :

- soit en temps réel : cas du spectrophotomètre de flamme utilisé pour des aérosols de $\mathrm{NaCl}$,

- soit en temps différé : cas de simples prélèvements sur filtres pour des aérosols fluorescents (sel de sodium de fluorescéine).

Afin de déterminer précisément le flux de la source contaminante (ø en $\mathrm{kg} . \mathrm{s}^{-1}$ ), indispensable pour accéder à la valeur des coefficients de transfert ( $k$ en $s . \mathrm{m}^{-3}$ ), le générateur dispose d'un moyen d'échantillonnage isocinétique. Rappelons que $\mathrm{k}=\mathrm{C} / \varnothing$.

\section{CARACTÉRISTIQUES TECHNIQUES}

- Fréquences de fonctionnement : 500 et $1800 \mathrm{kHz}$

- Dimensions des particules : diamètre aérodynamique médian en masse compris entre 0,3 et $5 \mu \mathrm{m}$

- Ecart-type géométrique de la dispersion granulométrique : 1,4 environ

- Débit de nébulisation de la solution à puissance maximale : pour $500 \mathrm{kHz}: 150 \mathrm{~cm}^{3} / \mathrm{h}$ pour $1800 \mathrm{kHz}: 300 \mathrm{~cm}^{3} / \mathrm{h}$

- Débit des aérosols secs : $1 \mathrm{mg} / \mathrm{h}$ à $10 \mathrm{~g} / \mathrm{h}$ selon la taille des particules

- Alimentation électrique : $220 \mathrm{~V} \pm 10 \%$

- Pression d'air : 3 bars 
- Remplissage, vidange et contrôle automatiques du niveau liquide

- Grande autonomie de fonctionnement

- Réglage d'air de séchage entre 3 et $18 \mathrm{~m}^{3} / \mathrm{h}$

- Puissance de génération réglable

- Régulation thermique de l'air de séchage.

\section{PRÉSENTATION}

Le générateur est constitué d'un boîtier et d'un clavier de contrôle. Tous les paramètres de fonctionnement sont réglés par le clavier. Un microprocesseur évite tout fonctionnement incorrect, simplifie l'utilisation du générateur par un menu défilant et assure un contrôle continu du fonctionnement. La chambre cylindrique qui entoure les deux pots de pulvérisation assure l'homogénéisation et le séchage des gouttelettes issues du pot en fonctionnement. Un couvercle conique permet un éventuel raccordement du générateur à une canalisation par l'intermédiaire d'un raccord rapide.

\section{DÉVELOPPEMENT ET COMMERCIALISATION}

Cet appareil a été développé à la suite de réflexions menées avec des spécialistes de la radioprotection du groupe CEA et avec le concours d'experts en physique des aérosols de I'IPSN. II bénéficie du savoir faire acquis sur le banc d'essais ICARE servant à l'étalonnage des moniteurs de contamination atmosphérique, banc dans lequel sont produits par le même procédé des aérosols radioactifs calibrés.

\section{Plusieurs appareils sont en fonctionnement au CEA.}

Le générateur "PLUS" est réalisé et commercialisé par la société SINAPTEC, 121, rue Chanzy à Hellemmes (59260) - Tél. : 20675905 Téléfax : 206759 95. S'adresser, pour tous renseignements, à M. Pascal TIERCE (SINAPTEC).

Sur le plan technique, vous pouvez également contacter au Centre d'études de Saclay (CEA/IPSN/DPEI): M. Jean CHARUAU (Tél. : (1) 6908 29 13). 